\title{
Analysis of Distributed System Strategy Research of Smooth Traffic in City
}

\author{
He LIN ${ }^{1, a}$, Ping-ji ZOU ${ }^{2, b}$, Hai-bo LI ${ }^{1, c}$, Yong-li LI, ${ }^{1, d}$ \\ ${ }^{1}$ School of information science and engineering, Lanzhou University, Lanzhou 730000, China \\ ${ }^{2}$ Department of information engineering, Lanzhou Vocational Technical College, Lanzhou 730070 , \\ China \\ alinhe88@163.com, bzpj0305@163.com, ${ }^{\mathrm{c} l i h b @ l z u . e d u . c n, ~}{ }^{\mathrm{d}} \mathrm{lyl} 168168 @$ sina.com
}

Keywords: urban traffic; road clear; distributed systems; urban roads; road jams; resource scheduling

Abstract. Principle and method of using a distributed computer system, to survey the current situation of urban traffic congestion, Discover urban road network structure topology and a distributed system with a certain degree of similarity, And the problems of urban traffic problems into computer. On urban road traffic condition and operation of distributed systems analyze the differences, given the distributed algorithm of urban road traffic flow scheduling, Change the 'illegal substitution penalty' promoted to 'blocking to unblock' strategy. Addressing the living environment and public waste of resources and low productivity caused traffic jams, such as restricting the development of city social problems, Enable distributed systems to ease urban traffic plays an important role, and ensure the smooth and efficient urban transport is the quality of urban life.

\section{Introduction}

City is main part of human civilization, Traffic is divided into three forms of land, sea and air transport. Land refers to walking on the ground transport of transport, cars, trains, etc. However, with the rapid development of urbanization and urban development urban transport, water resources and environment one of the three major problems of urban traffic problem in China is becoming more and more prominent [1]. To this end, the competent government departments, industry experts and community analysis of the causes of urban traffic, Proposed urban transport faces major problems and reason comes down to the following five areas: (1) the serious shortage of road capacity, (2) car was expanding too fast, (3) public transport keeps shrinking, (4) the low level of traffic management technology; (5) lack of overall transport strategy [2]. Now, government departments are given the appropriate solutions and strategies, but traffic congestion is still very serious.

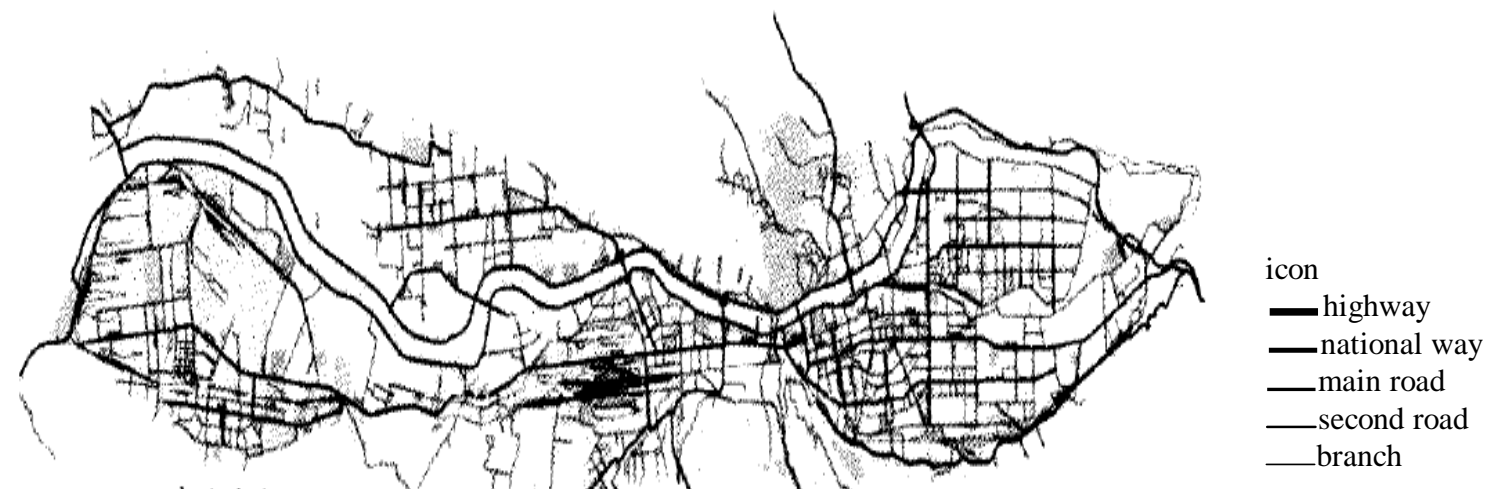

Fig.1 Road conditions in Lanzhou city

In this paper, is located in the geographical center of China, the Yellow River through the city, resident population of 3.6 million of cities-Lanzhou [3] as a sample, as shown in Figure 1. Traffic has also been 'From East to West is very crowded, From South to North is not smooth' embarrassed and confused, despite taking a cab control, vehicle tail number limit line, road and bridge construction and the construction of public transport and many other measures, take the total number of taxis control, 
according to the vehicle's tail number limit access, quicken the construction of public transport and increased construction of roads and bridges and other measures, but traffic jams still cannot be ignored. To this end, in 2012, the Lanzhou municipal people's Government, invited Prof. Zhou Ganshi academician of Chinese Academy of Sciences and Chinese Academy of engineering, the Ministry of public security and the Ministry of construction traffic 'smooth traffic project' Deputy team leader Yang Xiaoguang, United States Institute for transport policy development, Executive Vice President, green sustainable transportation advocates Kaer-Fei more than 30 experts, officials and ordinary citizens, taxi drivers, and representatives were invited to participate in the demonstration meeting, Lanzhou traffic jam problem, solutions and future Green transport planning to speak their minds, built to make suggestions and 'pulse' of Lanzhou city, the 'smooth traffic project'; Established the '12th five-year plan' and 'free traffic' plan, accelerate the construction of modern, open and intelligent traffic systems, congestion improved significantly in a relatively short time. As a computer worker, whether ideas in distributed computer systems in Lanzhou, poor transport, from the other side to help the Government to solve the traffic congestion problem.

\section{The similarity of Distributed systems and the urban roads}

\section{The concept of distributed systems}

Guarantee normal operation of the computer operating system. It is the soul of the computer system, is a collection of modules-to maximize the efficient and rational way to organize and manage computer resources, rational organization of computer workflow, the implementation of control procedures and provide a variety of services to the user, allowing users the flexibility, convenience, efficient use of computer, can make the whole computer system to run efficiently. Distributed operating system (DOS) based on a global approach to the management of system resources, it can be for any user to schedule network resource and scheduling process is 'transparent'. When a user submits a job, distributed operating system to choose the system that is most suitable processor, the user job submission to the handler, when the processor after completing the job, it will pass the results to the user. In this process, the user will not be aware of the existence of multiple processors, as well as a processor. Distributed software systems (DSS) is software to support distributed processing systems are interconnected by communication networks on a multiprocessor architecture mission system. Sometimes we call this software system distributed system (DS) [4], as shown in Figure 2.

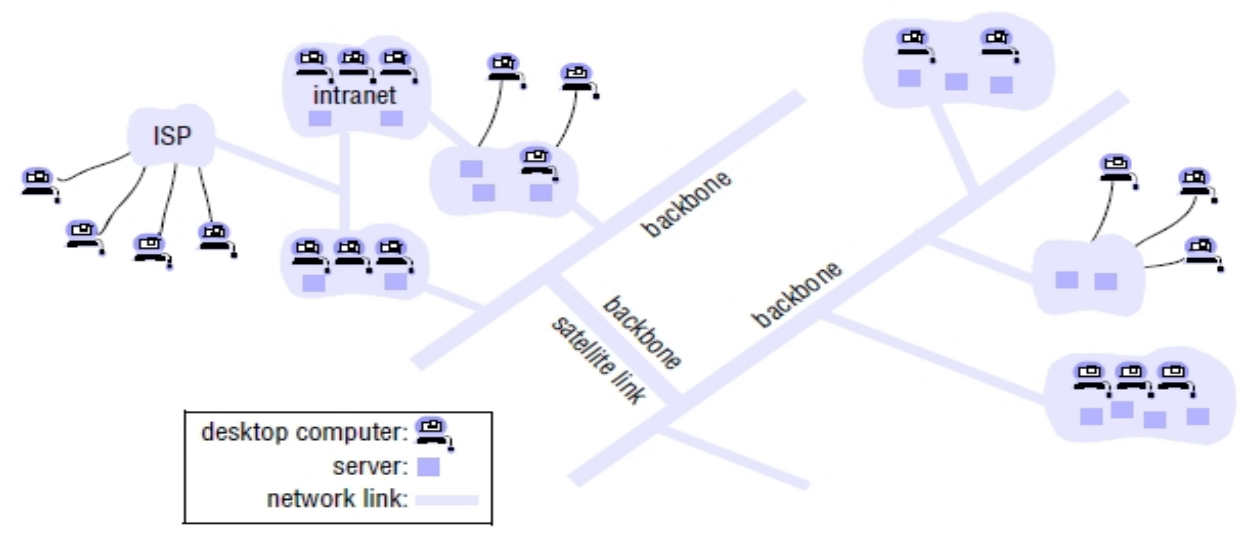

Fig.2 Distributed system topology

\section{Structure of the urban road system}

\section{(1) Road and city road}

Road is highway and urban road vehicle access with the jurisdiction of the unit allows community where, including the Plaza, public parks and other places for public access [5]. 
City road is a planned urban area for vehicles and pedestrians, with certain specifications for road, bridge and affiliated facilities, including roadways, sidewalks, squares, car parks, isolation belt, flyovers, pedestrian bridges, underpasses, river-crossing bridges, tunnels and other structures and expropriation of land for road construction within the planned red line [5].

\section{(2) Section and intersection}

Urban road transportation is divided into sections and intersections in two parts.

Sections are an essential part of the road, traffic on the section from the shunt, confluence and interweaving effect of vehicle running characteristics for continuous flow [5].

Two or more than two roads intersect in the same plane form intersections, level crossings are divided into light control and no light control control type [5].

\section{(3) The urban traffic system}

Urban traffic system by transport system (traffic operations), road system (channel) and traffic management system (management and control) consists of three parts [6]. Describe the three parameters of traffic on the roads: speed $V$, the flow rate $Q$, density $d ; d=Q / V$.

\section{From the urban road network structure has similarity with distributed system topology}

We compare Figure 1 and Figure 2, see distributed system topology structures and roads have many similarities in Lanzhou, According to the analysis, we found that there is a correlation between the two, so we give will be a distributed system model correlated with urban traffic model comparison table, see table 1.

Table 1 Distributed system model and correlation of urban traffic model comparison chart

\begin{tabular}{c|c|c}
\hline serial number & Distributed system & Urban traffic \\
\hline 1 & Communication channel & Road \\
\hline 2 & Process & Vehicle \\
\hline 3 & Scheduling algorithm & Traffic flow \\
\hline 4 & Network backbone & Main roads \\
\hline 5 & Internet service provider & Scheduling hub \\
\hline 6 & Communication protocol & Traffic laws \\
\hline 7 & Information transmission capacity & Road transport capacity \\
\hline 8 & Network failure & Traffic jams \\
\hline
\end{tabular}

According to table 1 we can see that in a distributed system is the core content of and constitutes a core element of city traffic can correspond to each other, By contrast we found that they have a great deal of similarity and relevance. Might as well let we try to construct a model that can satisfy this relationship.

\section{(1) The drive rate model of information in distributed systems}

Factors that affect distributed system process number, channel level of congestion, server capacity, and so on, we can construct information source for the drive rate depending on many factors as follows:

$$
d(t)=f\left(x_{1}, x_{2}, x_{3}, \ldots, x_{\mathrm{n}}, t\right)
$$

Where: $x_{1}$ is the communication channel, $x_{2}$ is the process, $x_{3}$ is the network backbone, and $x_{4}$ is an Internet service provider (ISP)....

The driving rate of information is defined per unit of time on the network through the number of processes or threads. Unit processes-threads per second. 


\section{(2) The drive rate model of traffic in urban road}

Influencing factors of urban traffic: the number of vehicles, the bearing capacity of roads, transportation network system, and so on, Many factors we can construct of urban road traffic in the drive rates are as follows:

$$
c(t)=g\left(y_{1}, y_{2}, y_{3}, \ldots, y_{\mathrm{n}}, t\right)
$$

Where: $y_{1}$ is the way, $y_{2}$ is the vehicle, $y_{3}$ is main road, $y_{4}$ is dispatching hub. ...

The driving rate of traffic is defined per unit of time on the road by vehicles (cars, etc). Unit vehicle per second.

Through the comparative analysis of these two models, combined application of distributed systems on a computer system so that normal operation of the system, use this method in urban traffic, and the evolution of the urban traffic problem into computer-related problems, so as to solve road congestion, free transport for the Government to provide a new approach and decision-making.

To this end, According to topology and algorithm model and variable with similarity analysis of relationship they have similarities, so we can take advantage of the similarities between them, to become an independent entity in distributed system already know, can work together to solve problems which cannot be solved by any single entity, corresponding to urban traffic problems, to solve road congestion.

\section{Urban road traffic condition and distributed system has differences of view}

Faced with the growing problem of urban traffic problems, foreign and domestic government authorities, experts, scholars and public views on the causes of congestion are analyzed. Construction departments mainly car road less irrational structure of the network, and a serious shortage of parking facilities; Police and traffic departments of traffic participants of weak legal system, poor civic awareness, poor technical condition of vehicles; There are also departments of urban traffic management in place, managing low level [2]. To this end, Countries, cities, departments put forward the corresponding countermeasures from different perspectives and recommendations.

However, traffic jam occurs in Lanzhou 'cause' where is it?

After investigation, we give the Lanzhou traffic problems and causes.

(1) The pedestrian system is not perfect

Small number of channels and bridges crossing the street, crossing the channel planning and location unreasonable or incomplete facilities, street lights lack. These phenomena causing the car not to let people, people without refuge; pedestrians only to cross the road, pass rates of the affected vehicles; pedestrians randomly across the street, competing with the vehicle road. This will be mapped to the table 1.-(7).

\section{(2) Road into the parking lot}

Planning and building owed a lot in the parking lot, serious shortfall in the number of public car parks and parking garages, there is no parking or parking on the road tour or on-street parking, misplaced make roads narrower and stop crowding, which disturbed the normal order of traffic and parking, to some extent, increased road congestion. This will be mapped to the table 1.-(8).

(3) The unreasonable design of bus lines and dispatching Center

Considerable part of the bus lines are concentrated in urban centers, in Xijindong Rd. more than more than 20 bus lines, transit network less densely populated outside of downtown. Clustered queues, 
the bus was dubbed by people 'strings'. Xiguan set at the throat of the dispatch Center, resulting in 'blocking add block' scene. This will be mapped to the table 1.-(3,5).

Traffic jams caused by more than three, is not an isolated problem in Lanzhou, but around a common phenomenon in the urban development. Stuck in traffic, not only affect drivers, also affects the work and life of the people; Stuck in traffic, not only adding time consumption, will cause more anxiety and shock. Stuck in traffic has made the urban road traffic in General drove rates seriously reduced, reduced the passage of vehicles, motor vehicles in urban areas but only $8 \sim 10 \mathrm{~km}$ per hour. Bike speed of $15 \mathrm{~km}$ per hour, faster by bike than by car, is not a joke, but a real life scene.

\section{Implementation of distributed urban road traffic scheduling algorithm}

According to the given for the drive rate model and the idea of distributed systems, as well as urban road traffic condition and difference analysis of distributed system operation, we distributed urban road traffic scheduling algorithm, algorithm design is shown in table 2.

Table 2 Distributed scheduling algorithm for urban road traffic

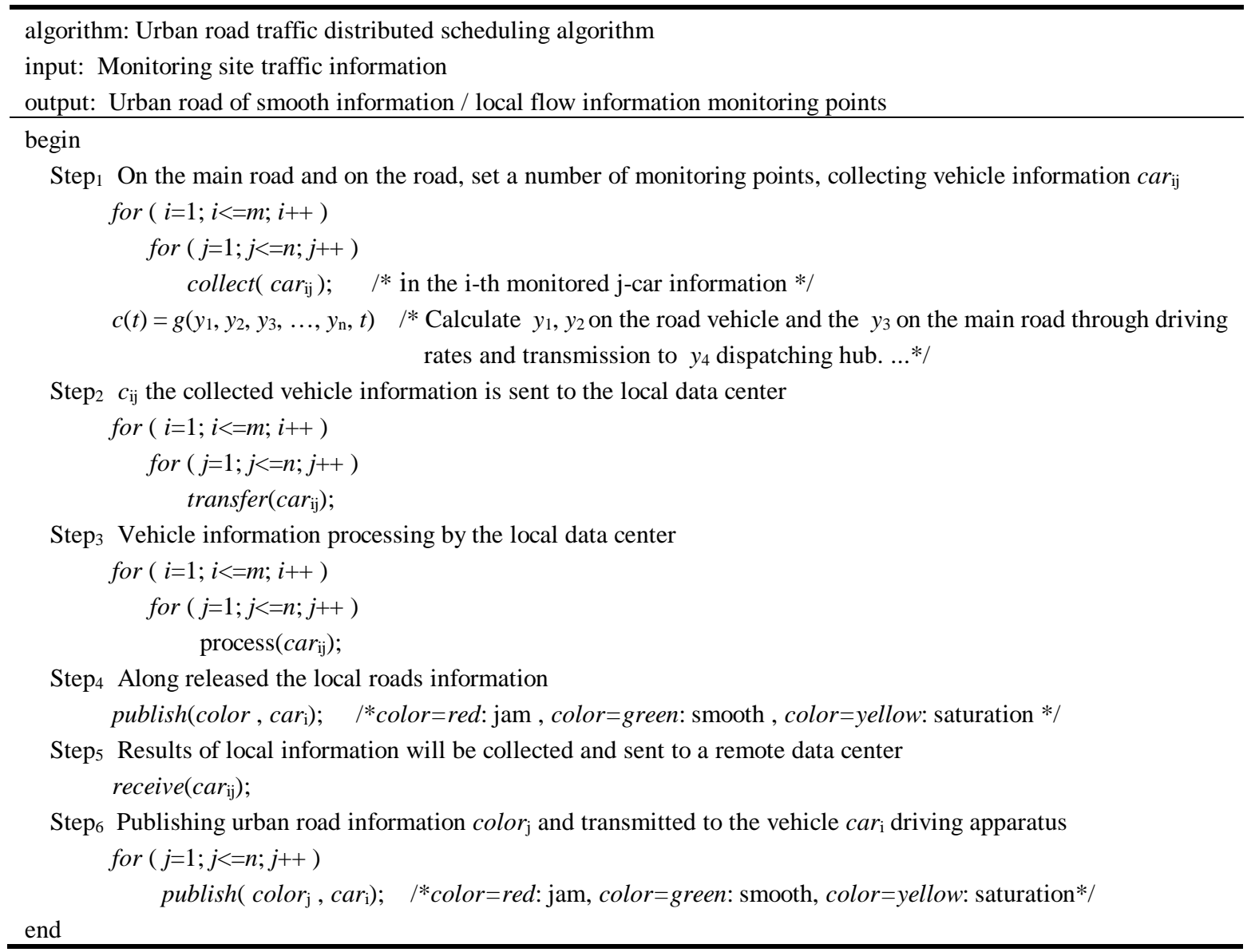

In this way, without adding equipment cases, expanding police functions, load the vehicle information and statistics software with distributed traffic scheduling algorithm of urban road, given the distributed algorithm of urban road traffic flow scheduling, Change the 'illegal substitution penalty' promoted to 'blocking to unblock' strategy. distributed computer systems of ideas really work, solve traffic congestion.

\section{Attach to the distributed system to alleviate traffic congestion}

Alleviating traffic congestion, it should abandon the original investment, strengthen the construction of road systems, improve the road network construction cycle. Strengthen the propaganda and education 
of traffic law, attention to ideological application of distributed computer system, improving urban management and service level.

$\mathrm{n}$ Improve the pedestrian system, equivalent to exclude networks in a distributed system model, at intersections with overpasses and underground passages.

$\mathrm{n}$ Government planning and building parking lots, equivalent to set up distributed system model of the communication channel, canceled stop lying, guaranteed access, open government compound in the parking lot.

$\mathrm{n}$ Play to passenger central role, equivalent to build a distributed system model of the Internet service provider, bus and passenger and subway is reasonable layout, highlight distributed system, improve the utilization of land.

$\mathrm{n}$ Electronic police role, equivalent to protection distributed system model of the process in the communication channel with the network (backbone) information on transmission capacity, on the road to increase electronic police numbers and traffic information icon, radical behavior of traffic violations, roads are clear.

Advocating the government's 'access limits forbid' policy, moderately increasing rental and car number, raising the city's speed, a new city is now before the exhibition.

\section{Conclusions}

Traffic arteries of the city, it shall remain open and efficient operation. However congestion has become negative factors of urban efficiency to address without delay. Traditional management tools and management has been unable to meet the demand for urban transport, especially in urban road network under continuous overload and a lack of effective management tools, any contradictions and subtle deficiencies in the management of traffic flow will be highly saturated rapidly, and very likely to cause widespread traffic congestion or even traffic standstill. Information technology is solution city road traffic management problem of important means, full using computer distributed system of thought, construction intelligent traffic management system, improve traffic management of systemic and scientific, expand high-tech application of cover range, mining road resources, regulation traffic flow, ease car road contradictions, improve city network traffic run efficiency and on burst accident event of fast reaction and fast disposal, enhanced traffic anti-risk capacity of effective way, well city traffic smooth engineering.

\section{Acknowledgements}

This work was financially supported by the Lanzhou scientific and technological projects (2013-3-93).

\section{References}

[1] Thomson I, Bull A. Urban Tragic Congestion: Its Economical and Social Causes and Consequences [J]. CEPAL Review, 2002, 76: 105-116.

[2] ZHOU T M, YANG J H. Study on the Method to Solve the Urban Traffic Congestion [J]. Journal of Chinese People's Public Security University (Natural Science Edition), 2005, 43 (1): 98-100.

[3] LU Y P. Traffic congestion problem and countermeasure analysis of Lanzhou City [J]. Technology \& Economy in Areas of Communications, 2013, 15 (4): 105-109.

[4] George Coulouris, Jean Dollimore, Tim Kindberg, Gordon Blair. Distributed Systems-Concepts and Design (Fifth Edition) [M]. Publishing by Addison-Wesley, 2011.

[5] People's Republic of China Law on Road Traffic Safety[S]. Beijing: China Legal Publishing House, 2004.

[6] JIN Z F. Transportation Planning and Organization [M]. Beijing: Publishing House Chinese People's Public Security University, 2003. 